\title{
Critical behavior of the 3D-Ising model on a poissonian random lattice
}

\author{
F. W. S. Lima ${ }^{1}$, U. M. S. Costa ${ }^{2}$, and R. N. Costa Filho ${ }^{2}$ \\ ${ }^{1}$ Departamento de Física, Universidade Federal do Piauí, 57079-970 Teresina,Piauí, Brazil \\ ${ }^{2}$ Departamento de Física, Universidade Federal do Ceará, 60451-970 Fortaleza, Ceará, Brazil.
}

\begin{abstract}
The single-cluster Monte Carlo algorithm and the reweighting technique are used to simulate the 3D-ferromagnetic Ising model on three dimensional Voronoi-Delaunay lattices. It is assumed that the coupling factor $J$ varies with the distance $r$ between the first neighbors as $J(r) \propto e^{-a r}$, with $a \geq 0$. The critical exponents $\gamma / \nu, \beta / \nu$, and $\nu$ are calculated, and according to the present estimates for the critical exponents, we argue that this random system belongs to the same universality class of the pure three-dimensional ferromegnetic Ising model.
\end{abstract}

PACS numbers: 05.70.Ln, 05.50.+q, 75.40.Mg, 02.70.Lq

\section{INTRODUCTION}

The Lenz-Ising model has a vast number of applications ranging from solid-state physics to biology. It is the oldest and most simple model for cooperative behavior which shows spontaneous symmetry breaking [1, 2]. The symmetry breaking is very important feature of transitions that can take place between phases with different symmetry like solid-liquid and ferromagnetic systems. The ferromagnetic transition is an example of continuous phase transition where the spin orientation symmetry is broken in the ferromagnetic phase due to the formation of magnetic domains. Inside each domain, there is a magnetic field pointing in a fixed direction chosen spontaneously during the phase transition.

One of the main characteristics of a continuous transition is the existence of critical exponents in the behavior of physical properties near the critical temperature. For example, the heat capacity obeys a power law

$$
C \sim\left|T_{c}-T\right|^{-\alpha},
$$

where $\alpha$ is the critical exponent associated with the heat capacity. When $0<\alpha<1$, the heat capacity diverges at the transition temperature. This is the behavior in the 3-dimensional Ising model for ferromagnetic phase transition. A natural question that follows is whether the critical exponent is modified in the presence of impurities or a random media. In such systems the influence of quenched disorder can be classified by the Harris criterion $3,[4,[5,6]$ which predicts that a perturbation is relevant if $\alpha>0$, where $\alpha$ is the critical exponent of the specific heat of the ideal system. For $\alpha<0$ quenched disorder is not relevant, while no prediction can be made in the marginal case $\alpha=0$.

Several studies using Monte Carlo simulations have shown that for site-dilution and bond-dilution disorder is relevant for the 3D ferromagnetic Ising model. However, when considering connectivity disorder using a Voronoi-Delauney lattice in the Ising 3D model, Janke et.al. [7, 8, [9], have shown that this kind of disorder does not modify the critical exponents. This result contradicts the Harris criteria. The explanation is that the disorder in the lattice was weak and more time running simula- tions was necessary to obtain some meaningful shift from the critical exponents on a regular lattice.

In this piece of work we investigate a 3D ferromagnetic Ising model on a Voronoi lattice using the same algorithm as in Ref. 7. But here, besides the fact that the coordination number is random, we add an additional factor to the randomness of the Voronoi lattice. The coupling between the neighbors spins now depends on their relative distance $r_{i j}$ according to the expression

$$
J_{i j}=J_{o} e^{-a r_{i j}}
$$

where $J_{o}$ is a constant and e $a \geq 0$ enters the model as a parameter 10. When $a=0$ we have the same results as in Ref. [7], i.e., the randomness in the number of coordination does not change the critical exponents. We show here that the addition of the new variable $J(r)$ does not change the universality class of the Ising model.

\section{MODEL AND SIMULATIONS}

We consider the ferromagnetic Ising model in a three dimensional Delauney lattice where the coordination number varies locally and the coupling factor depends on the relative distance between the first neighbors according to $\mathrm{Eq} 2$ In order to build the lattice, we first distribute $N$ sites $x_{i}$ randomly in a cube with edge equal to unit. To each site $x_{i}$ a polyhedric Voronoi cell $c_{i}$ is associated. This cell contains all the points $x \neq x_{i}$ whose Euclidian distance to $d\left(x, x_{i}\right)$ is inferior to the distance $d\left(x, x_{i+j}\right)$. A dual lattice is then constructed joining the $x_{i}$ of each cell that has a common edge. Using such a construction, the lattice connectivity is random and has an average value equal to 15 .

The Hamiltonian to the ferromagnetic Ising model can be described as

$$
-K H=\sum_{<i j>} J_{i j} S_{i} S_{j}
$$

where $K=1 / K_{B} T, T$ is the temperature, $K_{B}$ is the Boltzmann constant, and the coupling factor $J_{i j}$ is a function defined by Eq2 The spin $S_{i}$ can have two values 


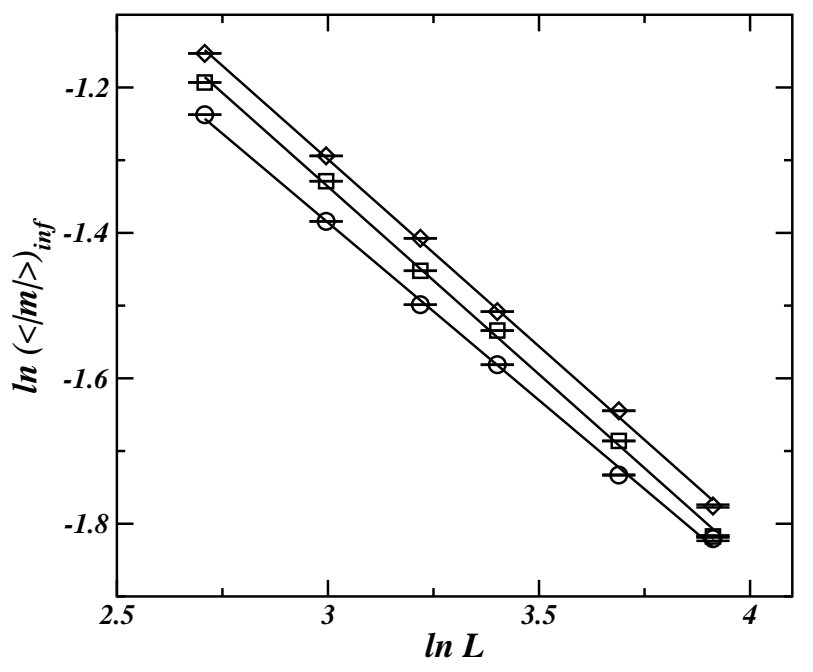

FIG. 1: Log-Log plot of the average magnetization $\langle|m|\rangle$ versus the system size $L$ for three different values of the coupling parameter $a$ : $a=0.0$ (circles), $a=0.5$ (squares), and $a=1.0$ (diamonds).

$S_{i}=1$ or $S_{i}=-1$, and the sum is evaluated in the first neighbors only. For simplicity, the system size used is defined in terms of a regular cubic lattice with side $L=N^{1 / 3}$. In our simulations we used several system sizes; $N=15^{3}, 20^{3}, 25^{3}, 30^{3}, 40^{3}$, and $50^{3}$. Depending on the system size, we have generated a different number of system copies: for $L=40$ and 50 we have done 25 copies; 30 copies for $L=30$, and 50 copies for $L=15$, 20 , and 25 . To perform the simulations we used the Wolf algorithm 11] close to the critical temperature $K_{c}$.

We start the simulations assigning to each site of the Voronoi lattice a random spin value, i.e. the initial state is totally random, and letting the system relax (reach equilibrium) for 120000 to 240000 timesteps or clusters. After that we generate from $2.4 \times 10^{6}$ to $4.8 \times 10^{6}$ clusters (depending on the system size) to evaluate the averages. For each 12 clusters the energy per spin $e=\frac{E}{N}$ and the magnetization per spin $m=\sum_{i} \frac{S_{i}}{N}$ were measured and registered on temporary files. In the end, we obtained from $2.0 \times 10^{5}$ to $4.0 \times 10^{5}$ values of the energy and magnetization which are is analyzed using the histogram technique 12]. In order to calculate the thermodynamic properties like specific heat $C^{(i)}(K)=K^{2} N\left(<e^{2}>\right.$ $-<e>^{2}$ ), for each copy classified by the index $i$ we used the reweighting technique to calculate the copies average indicated by the brackets in the expression below,

$$
C(K)=\left[C^{(i)}(K)\right] \equiv \frac{1}{R} \sum_{i}^{R} C^{(i)}(K)
$$

The variable $R$ means the number of copies in the simulations. The copies average is calculated over the the $C^{(i)}$ and not over the energy moments because the quenched averages must be calculated at free energy level instead of the partition function level. Finally, we determine the

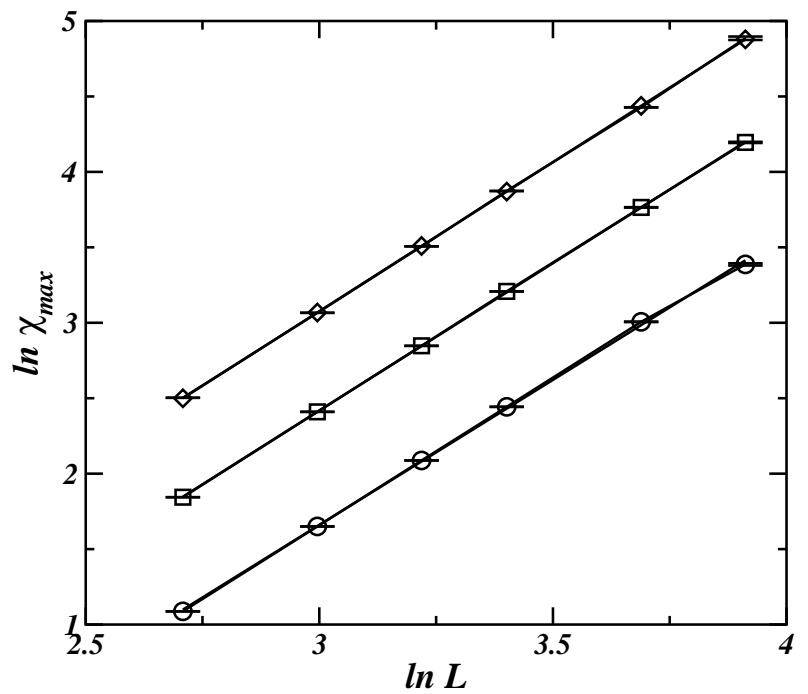

FIG. 2: The logarithm of the maximum susceptibility against the system size $L$ for $a=0.0, a=0.5$, and $a=1.0$.

maximum value $C_{\max }=C\left(K_{\max }\right)$ for each lattice size and analyze the scale behavior of $C_{\max }$ e $K_{C_{\max }}$ with the system size. Other thermodynamic quantities like susceptibility $\chi^{(i)}(K)=K N\left(<m^{2}>-<|m|>^{2}\right)$, magnetic cumulants $U_{2 p}^{(i)}(K)=1-\left(<m^{2 p}>/ 3<|m|^{p}>^{2}\right.$ ) $(p=1,2)$, and several derivatives related to $K$, such as $d^{(i)}<|m|>/ d K$, and $d^{(i)} l n<|m|^{p}>/ d K$, can be calculated in the same way. Once the dependence on the point $K_{0}$ is well known for each run through the reweighting, we can easily calculate the quenched averages of the quantities.

\section{RESULTS}

To estimate the critical temperature we calculated the magnetic cumulants $U_{2 p}$ using the histogram technique in combination with the finite size scaling analysis for three different values of the parameter $a$ that controls the strength of the coupling between the spins. For lattices sizes of $N \geq 8.000$ we get $K_{c} \approx 0.0724128$ and $U_{2}^{*} \approx 0.58567, K_{c} \approx 0.0724215$ and $U_{4}^{*} \approx 0.4645536$ for $a=0.0, K_{c} \approx 0.139265$ and $U_{2}^{*} \approx 0.58964, K_{c} \approx$ 0.139254 and $U_{4}^{*} \approx 0.46976$ for $a=0.5, K_{c} \approx 0.254941$ and $U_{2}^{*} \approx 0.587601, K_{c} \approx 0.254953$ and $U_{4}^{*} \approx 0.46765$ for $a=1.0$. These results show that the universal Binder cumulant $U_{2}^{*}$ is different from $U_{4}^{*}$ for each value of the parameter $a$ but they do not change for different values of $a$, and the critical temperature is the same for both values of the magnetic cumulant for each $a$. Also, the critical temperature decreases as the parameter $a$ increases. Such behavior is very similar to the one in dilution of pure systems.

To estimate the ratios of the exponents $\beta / \nu$ e $\gamma / \nu$, we use the fact that the magnetization on the inflexion point, 


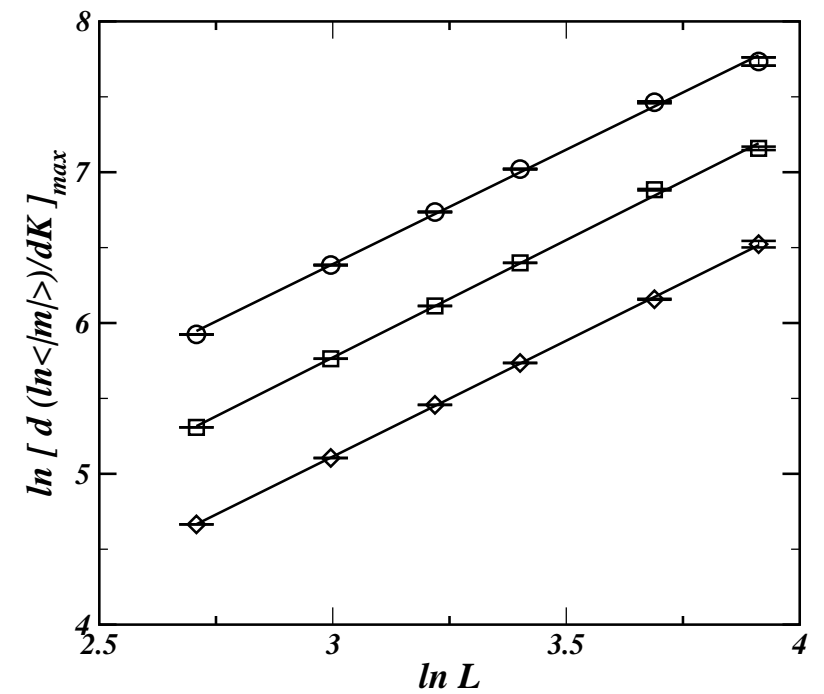

FIG. 3: Log-log plot of the maximum magnetization derivative against the system size $L$ for three different values of $a$ : $a=0.0$ (circles), $a=0.5$ (squares), and $a=1.0$ (diamonds).

and the susceptibility on $K_{\max }^{\chi}$ scales with

$$
\begin{gathered}
|<| m|>|_{\text {inf }}=L^{-\beta / \nu} f\left(t L^{1 / \nu}\right) \propto L^{-\beta / \nu} \\
\chi_{\max }^{\prime}(L)=\chi^{\prime}\left(K_{\max }^{\chi^{\prime}}(L), L\right)=A L^{\gamma / \nu},
\end{gathered}
$$

for large enough system sizes. Moreover, since the derivatives of the magnetization with respect to $K$ depends on the factor $L^{\gamma / \nu}$ which is an argument of the scale function $f$ [13],

$$
\left|\frac{d<|m|>}{d K}\right|_{\max }=L^{-\beta / \nu+1 / \nu} f^{\prime}\left(t L^{1 / \nu}\right) \propto L^{(1-\beta) \nu},
$$

the maxima of the logarithmic derivative scales like

$$
\left|\frac{d l n<|m|>}{d K}\right|_{\max }=\left|\frac{d<|m|>/ d K}{<|m|>}\right|_{\max } \propto L^{1 / \nu},
$$

and

$$
\left|\frac{d l n<m^{2}>}{d K}\right|_{\max }=\left|\frac{d<m^{2}>/ d K}{<m^{2}>}\right|_{\max } \propto L^{1 / \nu} .
$$

The above expression determines the value of the exponent $\nu$. Once finding that exponent, it is possible to calculate the values $\alpha / \nu$ of the specific heat. Through the finite size scaling analysis, it is possible to argue that the specific heat behaves like

$$
C_{\max }(L)=C\left[K_{\max }^{C}(l), l\right]=B_{0}+B_{1} \ln L
$$

in the second order phase transitions. To estimate the exponents ratio $\alpha / \nu$ we use the hiperscale relation $\alpha / \nu=$ $2 / \nu-D$.

In Figure (1) we show in a logarithmic scale a graph of the magnetization against the lattice size $L$ for three different values of the coupling parameter $a=0.0, a=0.5$,

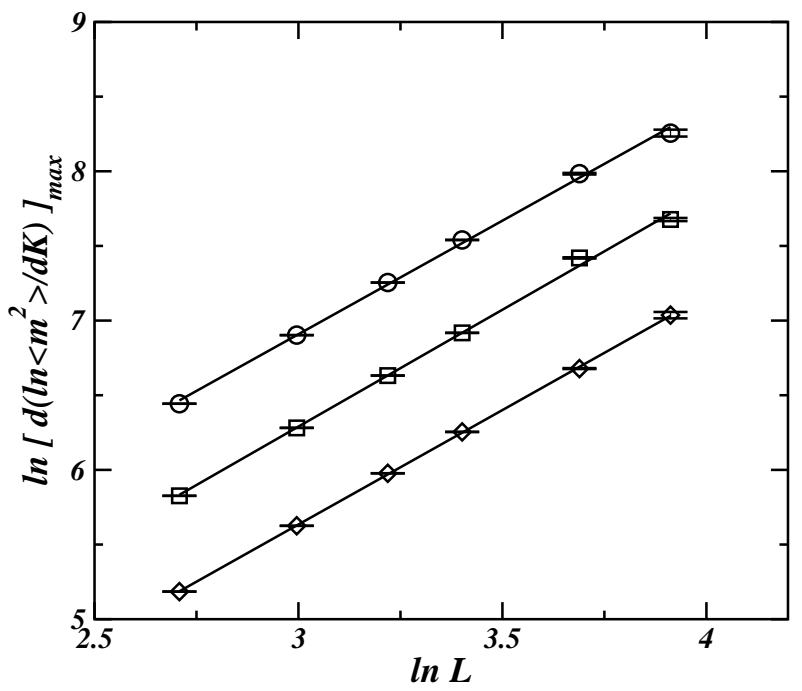

FIG. 4: Log-log plot of the maximum average squared magnetization derivative $(\mathrm{Eq}[9]$ against the system size $L$ for three different values of $a$ : $a=0.0$ (circles), $a=0.5$ (squares), and $a=1.0$ (diamonds).

and $a=1.0$. The linear adjustment of the square minima of the simulation data gives us $\beta / \nu=0.48920 \pm 0.00823$, $\beta / \nu=0.51628 \pm 0.00895$, and $\beta / \nu=0.51455 \pm 0.00683$, respectively. In Fig.(2) we plot the logarithm of the maximum susceptibility against the system size for $a=$ 0.0 (circles), $a=0.5$ (squares), and $a=1.0$ (diamonds). This curve inclination defines the exponents ratio $\gamma / \nu=1.9223 \pm 0.0295, \gamma / \nu=1.9537 \pm 0.0043$ and $\gamma / \nu=1.9758 \pm 0.0069$, respectively. Figs.(3) and (4) show a log-log plot of the maximum derivatives given by Eqs. 8] and 9] respectively, against the lattice size $L$ for $a=0.0, a=0.5$ e $a=1.0$. The linear fitting inclination gives $1 / \nu_{1}=1.5184 \pm 0.0302,1 / \nu_{1}=1.5581 \pm 0.0265$ and $1 / \nu_{1}=1.5364 \pm 0.0095$ and $1 / \nu_{2}=1.5207 \pm 0.0297$, $1 / \nu_{2}=1.5649 \pm 0.0332$ and $1 / \nu_{2}=1.5326 \pm 0.0078$, for the same values of $a$ as in the previous cases. In Fig. 5 we show the maximum value of the specific heat against the lattice size $L$ and through the hyperscale relation we get $\alpha / \nu \approx 0.0391,0.123$, and 0.069 , for $a=0.0,0.5$, and 1.0 respectively.

\section{CONCLUSION}

We have calculated here the critical exponents $\gamma / \nu$, $\beta / \nu$ e $\nu$ to the ferromagnetic Ising model in a three dimensional random Delauney lattice with a coupling factor depending exponentially on the distances between first-neighbors sites.

Dilution in a regular lattice was responsible for changing the universality class of this model, while random connectivity did not affect the exponents at all. The introduction of some kind of dilution through a distance dependent coupling factor in addition to the random con- 


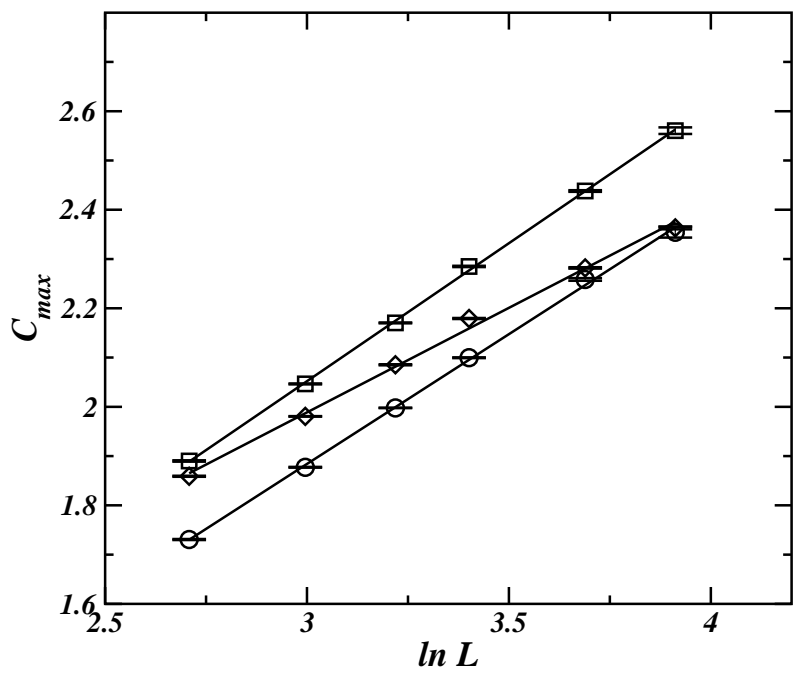

FIG. 5: The maximum of the specific heat against the lattice size for different values of the parameter $a$ as in the previous figures. nectivity of the lattice could increase the strength of the quench randomness and change the exponents. Although the critical temperature for the system decreases as the coupling factor $a$ increases indicating a dilution effect as in the case of a pure lattice, the results obtained here point out that these two ingredients are not enough to drive this system away from the universality class of the pure 3D ferromagnetic Ising model. That can be explained by the fact that instead of increase the randomness, the kind of dilution included here is responsible for the weakening of the long connections. This causes the lattice to become more regular and therefore the model is not affected by the effective randomness introduced.

\section{ACKNOWLEDGMENTS}

This work was supported by CNPq, FAPEPI and FAPEAL, Brazilian agencies.
[1] E. Ising, Z. Phys. 31, 253 (1925).

[2] H. N. V. Temperley, I. Syozi, in:C. Domb, J. Lebowitz(Eds.), Phase Transition and Critical Phenomena, Vol. 15, 1992, p.2.

[3] A. B. Harris, J. Phys. C 181671 (1974).

[4] S. Wiseman, E. Domany, Phys. Rev. Lett. 81, 22 (1998).

[5] S. Wiseman, E. Domany, Phys. Rev. E 58, 2938 (1998).

[6] H. G. Ballesteros, L. A. Fernández, V. Martín-Mayor, A. Muñoz Sudupe, G. Parisi, J. J. Ruiz-Lorenzo, Phys. Rev. B 58, 2740 (1998).

[7] W. Janke and R. Vilanova, Nuclear Phys. B 83, 697 (2000).
[8] W. Janke and R. Villanova, Phys. Rev. B 66, 134208 (2002).

[9] F. W. S. Lima, U. L. Fulco, and R. N. Costa Filho, Phys. Rev. E 71, 036105 (2005).

[10] F. W. S. Lima, J. E. Moreira, J. S. Andrade Jr., U. M. S. Costa, J. Phys. A 283, 100 (2000).

[11] U. Wolff, Phys. Rev. Lett. 62, 361 (1989).

[12] A. M. Ferrenberg, R. H. Swendsen, Phys. Rev. Lett. 61 2635 (1998); Phys. Rev. Lett. 63, 1658 (1989).

[13] W. Janke, Mohammad Katoot, R. Villanova, Phys. Rev. B 499644 (1994). 